\title{
Correction to: The Ecologies of Amateur Theatre
}

Correction to:

H. Nicholson et al., The Ecologies of Amateur Theatre, https://doi.org/10.1057/978-1-137-50810-2

The book was inadvertently published without the names of the chapter authors in the table of contents and the chapter opening pages. This has now been updated.

The updated online version of the book can be found at https://doi.org/10.1057/978-1-137-50810-2 